(C) 2021 Universidad Nacional Autónoma de México, Facultad de Estudios Superiores Zaragoza.

Este es un artículo Open Access bajo la licencia CC BY-NC-ND (http://creativecommons.org/licenses/by-nc-nd/4.0/).

TIP Revista Especializada en Ciencias Químico-Biológicas, 24: 1-11, 2021.

https://doi.org/10.22201/fesz.23958723e.2021.352

\title{
Panorama general de las adaptaciones metabólicas en el Orden Anura
}

\author{
David Ruiz-Londoño ${ }^{1}$, Santiago Manrique-Barros ${ }^{1 *}$ \\ y Luz Elena Triana Vidal ${ }^{1,2}$ \\ ${ }^{1}$ Programa de Biología, Facultad de Ingeniería y Ciencias, Pontificia \\ Universidad Javeriana. ${ }^{2}$ GRIEPIS, Programa de Medicina, Facultad de Ciencias \\ de la Salud, Universidad Libre seccional Cali, Santiago de Cali, Valle del \\ Cauca 760031, Colombia.*E-mail: smanrique2001@javerianacali.edu.co
}

\begin{abstract}
RESUMEN
En esta revisión se exponen de forma general las características del metabolismo del orden Anura (Fischer von Waldheim, 1813), reportadas desde mediados del siglo XX hasta la actualidad. Los Anura son calificados como organismos exitosos por poseer una amplia gama de mecanismos de adaptación a diferentes situaciones de estrés, posibles causas de la alta diversidad en este grupo. Se mencionan algunas adaptaciones en el proceso de asimilación de los carbohidratos con sus respectivos componentes fisiológicos, destacando la importancia de los corticosteroides en la activación de varias rutas metabólicas y la regulación de estos a través de factores como la temperatura y la estación anual. Se describen las principales funciones de los lípidos, las particularidades de las membranas y estrategias para eludir el estrés oxidativo, así como las adaptaciones del metabolismo de los compuestos nitrogenados y el almacenamiento de diversos alcaloides, la urea y su importancia en la osmorregulación posterior a la metamorfosis. Finalmente, se plantean perspectivas respecto a futuras investigaciones sobre la bioquímica de estos organismos.

Palabras clave: anuros, adaptaciones metabólicas, carbohidratos, proteínas, lípidos.
\end{abstract}

\section{Review of metabolic adaptations in Anura Order}

\begin{abstract}
This review article exposes the ecological characteristics of the Order Anura (Fischer von Waldheim, 1813) reported from mid-20th century to present, where they are described as successful organisms possessing a high range adaptation mechanism to different stress situations, which is considered as one of the possible causes for the high diversity in this group. Following, we describe some adaptations in the process of assimilation of carbohydrates and their respective physiological components are presented, highlighting the importance of corticosteroids in the activation of various metabolic routes and the regulation through factors, such as temperature and annual season. The main functions of lipids are described, which the particularities are emphasized in the formation of membranes and strategies to avoid oxidative stress. Subsequently, adaptations of the nitrogenous compound metabolism are explored where topics such as the storage of various alkaloids, urea and their importance in post-metamorphosis osmorregulation are evaluated as well. Lastly, there are prospects for future research on the biochemistry of these organisms.

Keywords: anurans, metabolic adaptations, carbohydrates, proteins, lipids.
\end{abstract}




\section{INTRODUCCIÓN}

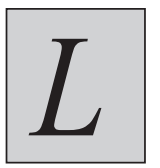

os anuros son un orden de anfibios con una gran diversidad morfológica y un peso corporal de menos de un gramo hasta varios kilos. La distribución geográfica de estos organismos abarca casi todas las regiones del planeta, exceptuando la Antártida; de gran variación de hábitat. forman un grupo taxonómico que alberga más de 7,301 especies (Frost, 2021; Wells \& Schwartz, 2007). La prevalencia de este grupo en hábitats tan diversos como bosques, desiertos, selvas, lagunas, lagos y estanques, que difieren ampliamente en factores como la temperatura, humedad, precipitación, salinidad, vegetación y fuentes de alimento; está correlacionada con los cambios morfológicos que les permiten adaptarse a diferentes ecosistemas (Citadini, Brandt, Williams \& Gomes, 2018). Los anuros en particular presentan características como vinculación al agua y a la humedad, ciclos de vida complejos con presencia de metamorfosis y sensibilidad fisiológica a condiciones ambientales a través de una piel con alta permeabilidad (Wake, 1991). Los anuros han sido modelo de investigación durante décadas por la facilidad tanto para encontrarlos como para manipularlos en el campo y el laboratorio en comparación con otros grupos de vertebrados (Amaya, Offield \& Grainger, 1998). Un ejemplo de los aportes generados de los estudios con este grupo es el descubrimiento del neurotransmisor acetilcolina a partir de la investigación realizada en los músculos de una rana (Flores \& Segura, 2005). Por lo anterior, se llevó a cabo la revisión de lo publicado desde mediados del siglo XX hasta la actualidad sobre los cambios y adaptaciones metabólicas que se han reportado en anuros a fin de exponer algunas de las adaptaciones del metabolismo de los anuros que se consideraron de mayor relevancia, en esta revisión. Sin embargo, es necesario mencionar que, aunque se tomaron en cuenta varios estudios de grupos taxonómicos puntuales, no es posible conocer si las conclusiones de estas investigaciones deben considerarse como generales para todo el orden.

Los anfibios han sido sujeto de comparaciones desde hace bastante tiempo por sus cualidades para adaptarse a condiciones de escasez de agua, alimento y oxígeno (Pough, 1980). Debido a esto, comprender la relación entre la bioquímica y la ecología de este grupo plantea un reto para los investigadores, quienes han abordado el tema desde un enfoque evolutivo. La clase Amphibia está compuesta por tres órdenes Anura(ranas y sapos), Caudata (ajolotes, salamandras y tritones) y Gymnophiona (cecilias) (Milner,1990).

Es pertinente tomar el inicio del nuevo milenio como referente, ya que resulta ser un hito para la evolución en el desarrollo de técnicas en la biología molecular, de gran relevancia como el verdadero auge de la reacción en cadena de la polimerasa (PCR), pese a haber sido desarrollada en 1983 por el Dr. Kary Mullis (Costa, 2004) y la culminación del proyecto genoma humano (PGH) en el año 2003 (Fernández \& Camargo, 2011)., Los hechos mencionados permiten compararlos con los descubrimientos de técnicas bioquímicas y las técnicas moleculares, mencionadas, por ser de gran ayuda en la comprobación de una hipótesis (Boto, 2010).

\section{Adaptaciones EN EL Metabolismo De LOS CARBOHIDRATOS}

El metabolismo de los carbohidratos en anuros no posee una gran variación con respecto al resto de la clase Amphibia, ésta a su vez, comparte gran cantidad de características con los mamíferos, lo que genera un especial interés en este grupo por realizar estudios que permitan comprender la evolución de las rutas metabólicas en otros vertebrados. Algunas de estas similitudes son la activación del proceso de degradación de azúcares, para la obtención de energía a través de señales químicas del sistema endocrino, donde el páncreas tiene un papel crucial en la producción de hormonas como la insulina y el glucagón (Cea-Calvo et al., 2008), así como el efecto de los inhibidores metabólicos sobre las enzimas que intervienen en el proceso de la respiración celular.

\section{Sistemas fisiológicos y la regulación del metabolismo de los carbohidratos}

En los anuros, existen tres sistemas fisiológicos que están encargados de regular el metabolismo de los carbohidratos: las hormonas de los islotes pancreáticos, los corticosteroides en la glándula hipófisis y finalmente el sistema de hormonas tiroideas en la glándula tiroides (McNabb, 1969). Por ejemplo, en anuros que habitan en zonas áridas y semiáridas (con periodos marcados de lluvia y sequía), como es el caso de Xenopus laevis, se ha observado que la degradación del glucógeno es regulada directamente por hormonas esteroideas, las cuales son altamente sensibles a los cambios de las condiciones ambientales como dieta disponible, temperatura y humedad. (Hanke \& Neumann, 1972). También se ha logrado establecer que en especies invasoras como Rhinella marina los altos niveles de glucocorticosteroides pueden ligarse con una alta tasa de dispersión, esto se encuentra regulado por un complejo sistema de interacción con la actividad locomotora, cambios de temperatura y factores de estrés, otras consecuencias de la presión ambiental sobre estos organismos se pueden explicar a través del eje hipotálamo-hipófisis-interrenal, como se observa en la Figura 1 (Brown, Shilton, Phillips \& Shine, 2007; Llewellyn, Thompson, Brown, Phillips \& Shine, 2012 ; Assis, Gardner, Smith, Gomes \& Mendonça, 2020).

Existen diversos métodos para medir la tasa metabólica estándar (TMS) en vertebrados, el más simple y utilizado en anuros es la calorimetría indirecta y el consumo de oxígeno (Lighton, 2008). El efecto de los cambios fisiológicos y ecológicos sobre este factor han sido estudiados durante décadas en los anfibios, así como en otros grupos con mecanismos de termorregulación similares (Glazier, 2005; Labra, Marquet \& Bozinovic, 2007). Sin embargo, investigaciones recientes han permitido añadir análisis filogenéticos, los cuales sugieren que las variaciones 


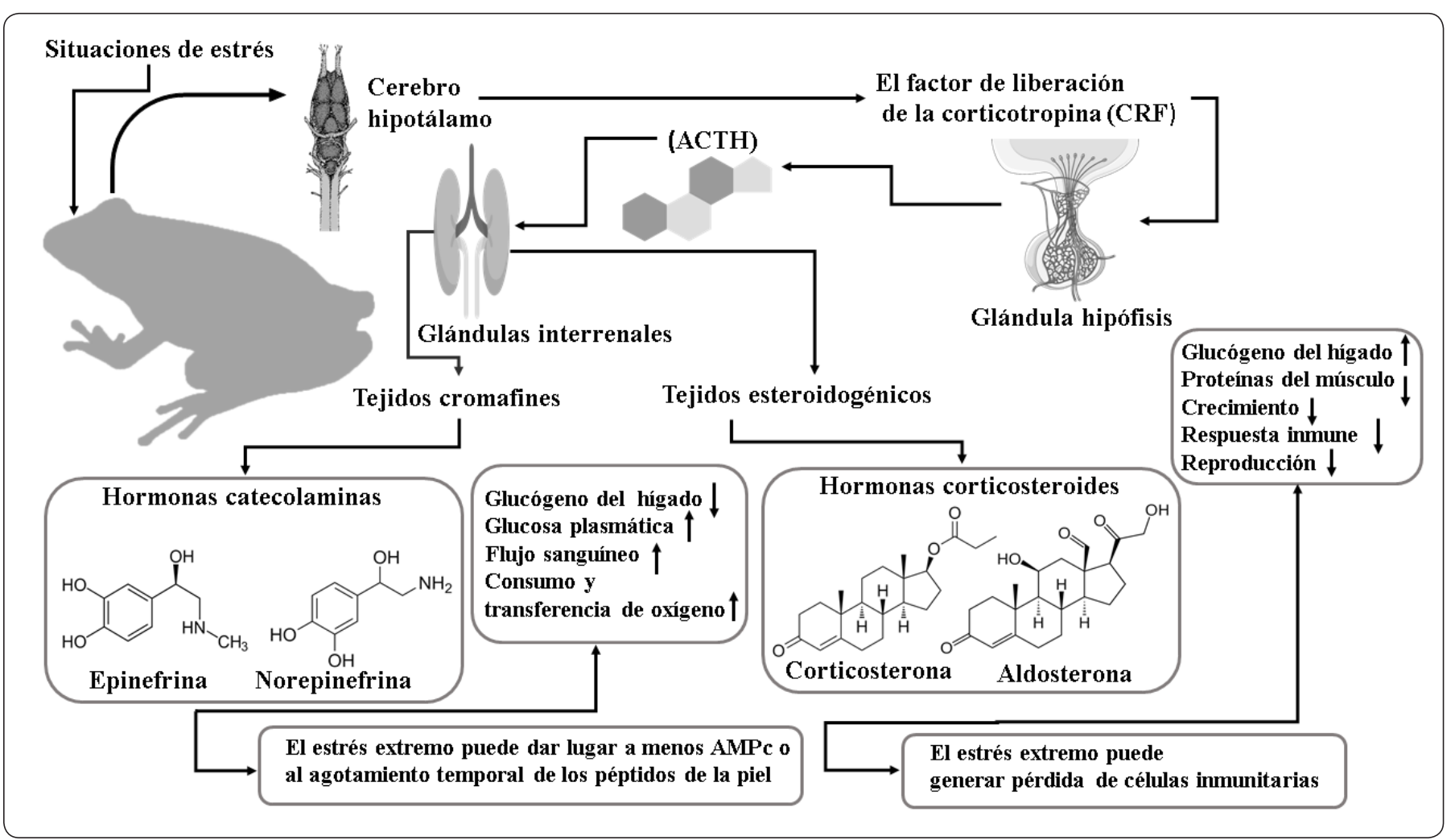

Figura 1. Diagrama esquemático del eje hipotálamo-hipófisis-interrenal (Eje HHI) que resume las principales reacciones en el organismo de los anfibios sometidos a condiciones de estrés como temperaturas extremas, desecación, infecciones por microorganismos entre otras reportadas por Denver (2009) y Rollins-Smith (2017). Estas situaciones generan la producción del factor liberador de la corticotropina (CRF) en el hipotálamo, que actúa sobre la glándula hipófisis. Esta segrega corticotropina, que es homóloga a la hormona adrenocorticotropa, transmitiendo la señal hacia las glándulas interrenales que cumplen las mismas funciones que la corteza suprarrenal en los mamíferos, donde se induce la producción de corticosteroides (aldosterona, corticosterona) en los tejidos esteroidogénicos y catecolaminas (epinefrina, norepinefrina) en los tejidos cromafines. Estas hormonas tienen efectos directos como reguladores del metabolismo de los carbohidratos e inducción de respuestas fisiológicas complejas que median procesos adaptativos importantes en el caso particular de los anuros. Modificado de Denver (2009).

de la (TMS) en la clase Amphibia son producto de la evolución fisiológica adaptativa y no permiten diferenciar a cada orden (Nespolo, Figueroa \& Solano-Iguaran, 2017). Lo anterior, es intrigante porque contrasta con los resultados de Chong \& Mueller (2013), quienes señalan a los anuros como poseedores de una (TMS) superior a la de las salamandras y cecilias.

Efectos de la temperatura en anuros y su relación con la glucosa

Durante los años que siguieron al 2000, los estudios sobre carbohidratos en anuros se enfocaron en determinar las adaptaciones que permiten la criopreservación en algunas especies, ya que se consideraba que la glucosa era un factor destacado para garantizar la supervivencia de estos organismos cuando se exponían a temperaturas por debajo de los $0^{\circ} \mathrm{C}$. Sin embargo, se desconocía si las reservas de la glucosa provenían del músculo o del hígado del animal. La investigación realizada por Steiner, Petenusci \& Brentegani (2000) logró determinar que es el glucógeno del hígado quien auxilia la falta de degradación de la glucosa producida por el frío, manteniéndose el glucógeno constante en los músculos. Pese a esto, puede no ser el único mecanismo responsable de la intolerancia a la congelación en grupos que carecen de esta adaptación.

El mecanismo de la glucosa como crioprotector generalmente funciona al reducir el punto de fusión de los fluidos corporales, disminuyendo la formación de cristales de hielo y el estrés osmótico relacionado a la temperatura del organismo. Además, estabiliza a las macromoléculas, protege a la membrana celular, y facilita otras respuestas fisiológicas al estrés por congelamiento, como la deshidratación de los órganos (Costanzo, Lee \& Wright, 1991). Cabe señalar que para que este proceso sea efectivo, la glucosa suele diluirse con otros compuestos químicos producto del metabolismo, como la urea, que se ha estudiado en Lithobates sylvaticus (MacDonald, Degenhardt, Baynes \& Storey, 2000).

La temperatura es una variable fundamental para comprender la variación en los niveles de glucosa en sangre y otros tejidos 
de especies de anuros adaptados a la criopreservación, así lo han reportado varios autores desde finales del siglo XX (Layne \& Lee, 1987; Costanzo, Wright \& Lee, 1992; Dinsmore \& Swanson, 2008). Además, se conoce que importantes reguladores del metabolismo de los carbohidratos en anuros como son los corticosteroides varían con la temperatura a través de diferentes épocas del año (Hanke \& Neumann, 1972). Esto se evidencia en otras investigaciones como la que fue llevada a cabo por Rodríguez, Bustos \& Sanabria (2019) donde se logró determinar que la concentración de la glucosa plasmática en Rhinella spinulosa tiene un aumento drástico cuando las temperaturas anuales oscilan entre los 0 y los $-1,6^{\circ} \mathrm{C}$.

Se ha reportado un aumento en las enzimas que median procesos como la glucólisis, el ciclo de Krebs y la cadena respiratoria, disminución significativa de los electrolitos, aumento de la glucemia, niveles bajos de hematocrito, eritrocitos y la hemoglobina durante la etapa de invierno en anuros como Lithobates catesbeianus, especie que ha servido como modelo biológico para entender los cambios fisiológicos y metabólicos de factores como la temperatura, la alimentación y el crecimiento en anuros (Coppo, Mussart, Fioranelli, Barboza \& Koza, 2005).

A pesar de las adaptaciones desarrolladas para tolerar los cambios en la temperatura, se describió que diferentes especies tienen una selectividad térmica para potenciar el retorno energético, mientras que al realizar los procesos de ingestión del alimento, digestión, absorción y asimilación por encima de estas preferencias el proceso ocurre más rápido, pero con un menor rendimiento energético, como se estudió en Leptodactylus latrans (Timpone, Gavira \& Andrade, 2019)

\section{AdAPTACIONES METABÓLICAS de LOS LíPIDOS}

En Colombia, diversas investigaciones se han encargado de relacionar la diversidad y distribución de las comunidades de los anuros en zonas con estacionalidad térmica y pluviométrica (Rincón \& Castro, 1998; Vargas \& Castro,1999). En gran parte de las especies adaptadas a los cambios abióticos abruptos se ve una notoria influencia en su comportamiento alimenticio y reproductivo en periodos cálidos con detrimento de la tasa metabólica en los fríos (López, Scarabotti \& Ghirardi, 2011). Durante el tiempo que disminuye la temperatura se ha demostrado que los cuerpos grasos compuestos por moléculas lipídicas son una de las reservas energéticas más importantes en este grupo (Prado \& Hadad, 2005; Wells \& Schwartz, 2007). De esta forma, se puede dilucidar el papel que tienen estos compuestos en el metabolismo de los anuros, así como en otros grupos de vertebrados. Sus funciones van desde reservas energéticas en actividades de alto gasto energético como la vocalización, la cual es una actividad de suprema importancia para el comportamiento y comunicación de los anuros, donde los lípidos contribuyen del 75\% al 90\% (Bevier, 1997; Grafe, 1997) de energía en algunas especies, también son parte indispensable de las membranas biológicas (fosfolípidos, esfingolípidos y colesterol), además de servir como esqueleto de diversas moléculas con funciones de señalización por ejemplo, los corticosteroides y las prostaglandinas, finalmente pueden cumplir funciones vitamínicas que se encargan de proteger a los organismos del estrés oxidativo (McKee \& McKee, 2014).

\section{La metamorfosis con relación al metabolismo de los lípidos} Gran parte de los vertebrados se adaptan a un tipo específico de hábitat, por lo que conservan un mismo estilo de vida después del nacimiento. No obstante, los cambios fisiológicos y morfológicos que presentan los anuros a causa de la metamorfosis implican la alteración de estas dinámicas durante las diferentes etapas del desarrollo (Dickhoff, 1993). El proceso de la metamorfosis es posible gracias a la regulación coordinada de todos los aspectos del metabolismo de un organismo, incluyendo las biomoléculas de reserva energética como los lípidos. (Sheridan, 1994).

Una estrategia común en el metabolismo de los lípidos es la presencia de una primera fase de acumulación en los sitios de almacenamiento, esto es producto de la lipogénesis que prima sobre la lipólisis. Por el contrario, la segunda fase se caracteriza por la disminución de las reservas lipídicas con un aumento considerable de la lipólisis sobre la lipogénesis. Estos procesos de regulación y movilización aseguran la disponibilidad de lípidos en momentos críticos, además obedecen a diferentes estímulos internos como la interacción entre hormonas y externos como la temperatura y la humedad entre otros (Sheridan \& Kao, 1998).

Numerosos autores se han encargado de estudiar la importancia de la dieta sobre la metamorfosis en anuros desde diferentes enfoques como la ecología, fisiología, biología del desarrollo y la bioquímica, donde se ha podido establecer que a pesar de que la mayoría de los renacuajos son considerados herbívoros, existen diversas especies con hábitos de canibalismo, ovofagia, coprofagia y depredación, los renacuajos de diversas especies crecen y se desarrollan más rápidamente cuando hay un alto consumo de proteínas.

Este aumento en la ingesta de proteínas en la dieta, permitió evolutivamente que la función del sistema tiroideo se especializara en regulaciones metabólicas y fisiológicas; por ejemplo la transformación de la tiroxina (T4) a, 3,5,3'triyodotironina $(\mathrm{T}$, ) induce el ciclo de metamorfosis en anfibios, esta transformación depende de la disponibilidad de la proteína en el hábitat (Wynne \& Bold, 1985). Las principales fuentes alimenticias de los renacuajos suelen ser algas filamentosas verdes y diatomeas, estas últimas almacenan el exceso de fotosintatos en forma de lípidos, aparte de ser ricas en proteínas debido a su estado de simbiosis con cianobacterias fijadoras de nitrógeno (Wynne \& Bold, 1985), los renacuajos que tienen esta dieta se desarrollan más rápido y alcanzan mayores tamaños (Kupferberg, 1997). Se debe resaltar que pese a la existencia de larvas (renacuajos) que tienen un alto requerimiento proteico y lipídico, las fuentes nutricionales y 
hábitos de forrajeo son muy distintos a los del adulto, lo que representa una ventaja ecológica, ya que se evita la competencia entre larvas y adultos.

Los estudios enfocados en dilucidar los mecanismos bioquímicos relacionados con la metamorfosis en anuros, han encontrado un aumento de las hormonas tiroideas como la: (Weber, Farrar, Tom \& Grau, 1994), somatostatina (King \& Millar, 1981), prolactina, hormona del crecimiento, aldosterona, corticosterona (Jolivet-Jaudet \& Leloup-Hatey, 1984; Kikuyama, Suzuki \& Iwamuro, 1986), hormona liberadora de la tirotropina (King \& Millar, 1981; Balls, Clothier, Rowles, Kiteley \& Bennett, 1985) y una disminución en los niveles de insulina. Todos estos hallazgos se acompañan de una compleja regulación fisiológica durante el proceso de la metamorfosis, que tiene un efecto directo o indirecto sobre los cambios en el metabolismo de los lípidos, que es especialmente relevante en la obtención de moléculas como el colesterol, que sirve como precursor de las hormonas, que a su vez están involucradas en los cambios del metabolismo de los carbohidratos durante la metamorfosis. La interacción entre los factores bioquímicos y fisiológicos en el metabolismo de los lípidos con efectos sobre la metamorfosis se puede observar en la Figura 2.

\section{Adaptación homeoviscosa}

La aclimatación se refiere a los cambios fisiológicos o de comportamiento que ocurren en un organismo en respuesta al estrés causado por cambios en los factores climáticos en ambientes controlados, como la temperatura (Hart, 1957). Esta característica se convierte en un rasgo crucial para que los organismos puedan colonizar diferentes nichos.

Ceballos (1999) investigó las biosíntesis de la aldosterona en los anuros, donde trató temas relacionados con las adaptaciones metabólicas a los cambios de temperatura, se presumía que estos poseían la propiedad homeoviscosa, que consiste de forma superficial en la capacidad de someter los enlaces entre carbonos y las enzimas de la membrana celular a cambios conformacionales, logrando modificar los puntos de fusión de los fosfolípidos en la membrana celular, manteniéndola en un estado de fluido bajo casi cualquier cambio térmico permitiéndole seguir con sus funciones biológicas corrientes

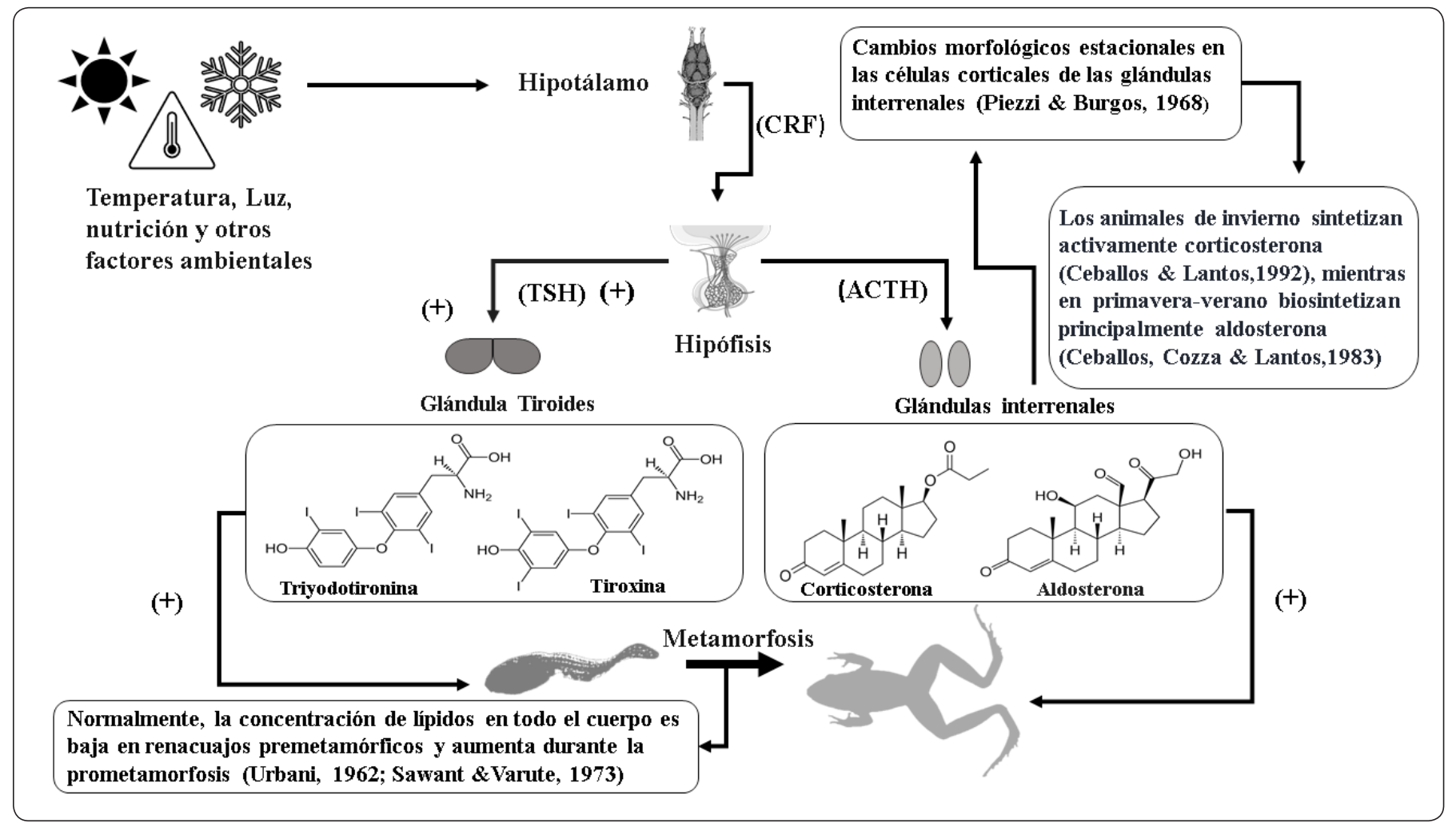

Figura 2. Diagrama del eje hipotálamo-hipófisis-tiroides con algunos de los estudios que reportan influencia de este sobre el metabolismo de los lípidos. Los factores ambientales estimulan el hipotálamo, este a su vez transmite la señal en forma del factor liberador de corticotropina (CRF) hasta la glándula hipófisis donde la producción de corticosteroides ocurre de igual forma que en la Figura 1. Simultáneamente la hipófisis produce la hormona estimulante de la tiroides (TSH) que genera la producción de tiroxina (T4) y triiodotironina (T3) en la glándula tiroides. Las hormonas tiroideas y los corticosteroides inducen la transformación de tejidos durante la metamorfosis, además de cambios importantes en todas las rutas metabólicas. Modificado de Tata (1999). 
como se había estudiado ampliamente en otros organismos como Escherichia coli (Sinensky, 1974), en Tetrahymena pyriformis (Kasai et al., 1976) y en peces (Cossins \& Prosser, 1978), debido al alcance de estudios previos no se logró conocer la importancia de la homeoviscocidad en los anfibios, pese a esto no se descarta la posibilidad de estudiar los cambios en la saturación de los lípidos como un sistema fisiológico-bioquímico para la aclimatación en poiquilotermos.

\section{Limitación de las reservas lipídicas en actividades extenuantes}

Los anuros se caracterizan por emitir una gran variedad de cantos y sonidos que conforman un sistema complejo de comportamiento y comunicación (Cocroft \& Ryan, 1995; Dapper, Baugh \& Ryan, 2011). La estructura de la señal acústica en anuros ha sido considerada única entre cada especie como una estrategia de aislamiento reproductivo (Gerhardt, 1994) y, por lo tanto, ha sido utilizada para el reconocimiento de nuevas especies crípticas (Heyer, García-Lopez \& Cardoso, 1996). A causa de esto, se ha decidido indagar sobre la fuente energética que sustenta este comportamiento en la familia Hylidae, esperando que fueran los cuerpos grasos quienes apoyaran esta labor que suele durar toda la noche, sin embargo, los resultados de Carvalho, Gomes \& Navas (2008), reflejan que no hay un cambio entre el contenido total de lípidos en el músculo del tronco y la tasa de llamadas entre diferentes especies, lo que podría deberse a diferentes aspectos metabólicos para prevenir la formación de cuerpos cetónicos dañinos como resultado del catabolismo de los ácidos grasos.

La característica de no hacer uso de las reservas lipídicas en formas de cuerpos grasos pese a realizar una acción con alto gasto energético, no está restringida al canto pues Antoniazzi, López, Duré \& Falico (2012) estudiaron la alimentación de dos especies de anfibios (Anura: Hylidae) en la estación de bajas temperaturas y su relación con la acumulación de energía, encontrando que la especie Hypsiboas pulchellus se caracterizaba por el poco desarrollo de los cuerpos grasos y el bajo índice de vacuidad gastrointestinal pese a tener un periodo de actividad muy prolongado que se extiende hasta las estaciones más frías.

\section{Adaptaciones en el Metabolismo de LOS AMINOÁCIDOS}

A diferencia de las rutas metabólicas como la glucólisis, la síntesis y degradación de ácidos grasos; la biosíntesis de compuestos nitrogenados no se ha mantenido tan conservada en los vertebrados. Ya que, la mayoría han perdido la capacidad para sintetizar todas las vitaminas y las cadenas carbonadas de aproximadamente la mitad de los aminoácidos, es decir los que son considerados esenciales (Zalduegui, 1976). No obstante, estas sustancias se pueden obtener a través de la digestión de las proteínas, esta comienza con el efecto de las amilasas y las lipasas que se hallan en la saliva, secretadas por las glándulas salivales, posteriormente cuando el alimento pasa al estómago, será sometido a procesos de digestión enzimática, desnaturalización e hidrólisis de las proteínas gracias a la acción del ácido clorhídrico, además de enzimas como las lipasas y las pepsinas. En el intestino se da la acción proteolítica de las enzimas pancreáticas, por la activación de tripsinógenos, propeptidasas y fosfolipasas para seguidamente realizar la absorción de los aminoácidos. Este es un proceso que se da de forma similar tanto en anfibios como en otros grupos incluidos los mamíferos (Martínez \& Martínez de Victoria, 2006).

\section{Captación de alcaloides y sus ventajas ecológicas}

Los anuros son un orden que resalta por su alta diversidad sobre otros grupos taxonómicos (Frost, 2021). La familia Dendrobatidae de ranas neotropicales ha sido objeto de estudio durante varios años debido a la presencia de alcaloides tóxicos, acompañada de una respuesta aposemática como mecanismo de defensa ante los depredadores, para esta familia en particular se ha demostrado la naturaleza exógena de sus alcaloides que son extraídos a través de una dieta específica de artrópodos (Darst, Menéndez Guerrero, Coloma \& Cannatella, 2005). Estas sustancias son descritas como un conjunto de compuestos orgánicos nitrogenados que derivan del metabolismo de los aminoácidos, son débilmente alcalinos, presentan una complejidad molecular moderada y generan alteraciones fisiológicas generalmente nocivas en los organismos (Daily, Myers \& Whittaker,1987).

Actualmente en los anfibios se han encontrado y descrito más de 800 tipos de alcaloides lipofílicos, además de 30 aminas biogénicas y 266 péptidos (Daily, Myers \& Whittaker, 1987; Proaño \& Rivera, 2005). Todos estos compuestos han demostrado utilidad no solo para evitar la depredación, sino también como herramienta para evitar la infección por microorganismos (Daily, Spande \& Garraffo, 2005) lo que los convierte en una posibilidad cada vez más grande de bioprospectos para la industria farmacéutica (Gutiérrez, Morales \& Pino, 2018.). Por lo tanto, conocer las características más relevantes del metabolismo de los aminoácidos en anuros podría ayudar a comprender las diferentes rutas metabólicas que les permiten acumular una gran variedad de moléculas tóxicas provenientes de otros organismos (Gómez Fernández, Castaño, Fierro, Armbrecht \& Asencio-Santofimio, 2013).

\section{El hígado de los anuros}

La mayoría de los tejidos en vertebrados tienen al menos una capacidad limitada para realizar algunos de los procesos del metabolismo de los aminoácidos. Sin embargo, el hígado es el componente esencial del metabolismo del nitrógeno e interviene en la regulación de los niveles de aminoácidos en sangre, es la principal reserva de glucógeno en actividades de alto consumo energético como la vocalización y la depuración de sustancias tóxicas. Algunos anuros como la rana Andinobates minutus 
(Dendrobatidae) han mostrado la particular eficacia del hígado, al presentar una menor producción de bilirrubina en comparación con otros órdenes, otorgándole una singular resistencia a la ictericia, pese a esto se desconoce la naturaleza de esta adaptación(Federico, 2011). Amedida que las relaciones filogenéticas se ramificaron entre caudados y anuros, estos últimos, desarrollaron un hígado con características histológicas idénticas a los mamíferos. Por otro lado, los órdenes Caudata y Gymnophionade poseen estructuras del tejido hematopoyético en la región perihepática subcapsular y en el campo portal, de las que carecen los anuros. Lo anterior podría dilucidar tanto diferencias fisiológicas como bioquímicas en el desarrollo del sistema inmunológico del bazo y la médula ósea (Akiyoshi \& Inoue, 2012).

\section{Compuestos nitrogenados en el mantenimiento de la homeostasis}

Los anuros adultos son animales ureotélicos como el resto de los anfibios, es decir, que excretan urea como resultado del exceso de nitrógeno, el ciclo de la urea es una característica de la que carecen en su estado larval y la adquieren durante la metamorfosis a través de la producción de hormonas en la hipófisis, que estimulan la expresión de genes que codifican para las enzimas que realizan este proceso (Grundy \& Storey, 1994). La aparición de la capacidad para sintetizar la urea se encuentra a la par con el desarrollo de adaptaciones que permiten su almacenamiento (Balinsky, 1981).

Aminoácidos libres en la regulación de fluidos intracelulares Una gran cantidad de anfibios tanto anuros como caudados rana, ranas Fejervarya cancrivora, Bufouiridis y Xenopus laevis se encuentran entre los organismos más estudiados con esta capacidad. Sucede que en respuesta a un ambiente salino, se observa aumento del sodio y cloruro en el plasma, además de aumento de potasio y cloruro intracelular, los aminoácidos libres contribuyen poco a la osmolaridad del plasma, pero son indispensables en la regulación de fluidos intracelulares, lo que contribuye a mantener el equilibrio de la presión osmótica de los fluidos corporales siempre que estos se mantengan a un nivel más alto que el entorno (Balinsky, 1981).

Los resultados de las investigaciones realizadas en anuros eurihalinos durante mediados de la década de los 60 y principios de los 80 describen el aumento significativo de los aminoácidos libres no esenciales en el músculo en respuesta al aumento de la salinidad, especialmente de glicina y alanina, también se encontró que en salinidades muy altas se presentaba un menor aumento de todos los aminoácidos libres en el plasma (Gordon, 1965; Baxter \& Ortiz, 1966; Colley, Rowe, Huggins, Elliott \& Dicker, 1972; Balinsky, Dicker \& Elliott, 1972). Esto refleja un aumento en el catabolismo proteico que genera nitrógeno para la síntesis de la urea, compuesto con una función directa en la osmorregulación del organismo (Balinsky et al., 1972).

\section{El papel de la urea en la osmorregulación}

La familia de proteínas de las acuaporinas cumple funciones como la apropiada concentración de orina, secreciones glandulares y reabsorción de fluidos, en anuros, estas también regulan las respuestas fisiológicas ante cambios en el ambiente, incluso durante la metamorfosis, además de cumplir un papel fundamental en la osmorregulación del organismo (Krane \& Goldstein, 2007). Se conoce que los anuros presentan hasta 6 clases de acuaporinas, de las que 2 son propias de este orden (Suzuki, Hasegawa, Ogushi \& Tanaka, 2007), siendo identificadas principalmente en piel, vejiga y grasa corporal (Ma, Baoxue \& Verkman, 1996; Virkki, Franke, Somieski \& Boron, 2002; Zimmerman et al., 2007). Lo anterior puede estar ligado con la capacidad que tienen algunas especies de anuros como Bufo cognatus en almacenar en su vejiga hasta el 30\% de su peso corporal en orina, previniendo la deshidratación y actuando como amortiguador en situaciones de estrés osmótico debido a la alta concentración de urea (Ruibal, 1962).

Posterior a la fase larval, la urea cobra un papel fundamental en la osmorregulación de los anuros de agua dulce como la Rana cancrivora, ya que estos presentan una relación hiperosmótica con respecto al medio, lo que puede generar sobrehidratación y pérdida de electrolitos que pasan al agua con una menor concentración de sales. La emisión de urea diluida en orina puede servir como una solución hipotónica que acompañada de procesos fisiológicos como la resorción tubular de sales y la absorción cutánea de electrolitos ayudarían a mantener la homeostasis en el organismo (Eckert, Randall \& Augustine, 1992).

Balinsky (1981) afirmó que no se conocía ningún anfibio con la capacidad de sobrevivir cuando su ambiente interno es hiposmótico diferente al entorno en el que vive, a excepción de los renacuajos de la Rana cancrivora. Cuando esta especie se encuentra en etapa adulta la sangre se mantiene hiperosmótica con respecto al medio, incluso en condiciones de alta salinidad, su orina posee solo un $10 \%$ de los elementos en el plasma, además de una menor concentración de sales y bajos niveles de urea, que evidencian la función del compuesto nitrogenado a mantener la hiperosmolaridad con el medio (Wilson, 1989).

\section{Conclusiones}

Los anuros, han demostrado ser un grupo exitoso, no solo por ser el orden de anfibios con el mayor número de especies, sino también por su amplia distribución en el planeta, ya que, gracias a su naturaleza eurihalina pueden llegar a colonizar desde ecosistemas acuáticos, tanto de agua dulce como de agua salada, hasta una amplia diversidad de ecosistemas terrestres. El fundamento de este éxito se debe mayoritariamente a las adaptaciones en su fisiología y metabolismo, que les permiten mantener la normalidad de sus procesos biológicos funcionales bajo escenarios desfavorables. Son un grupo de vertebrados 
especializados en situaciones de estrés térmico, desarrollando estrategias como la estivación, la capacidad de mantener un estilo de vida activo en estaciones frías, llegando incluso a poder realizar complejos sistemas de comunicación y reproducirse, acciones que requieren de mucha energía en este tipo de condiciones donde mantener una tasa metabólica elevada tiene un alto precio y significa una labor ardua para el organismo. Se considera que el estudio de las rutas metabólicas que permiten la captación de alcaloides, péptidos y aminas biogénicas es aún bastante limitado, por lo que se podría ahondar más en este tema y relacionarlo con la ecología de estos organismos. Por otra parte, las investigaciones relacionadas con los lípidos y carbohidratos son más abundantes y recientes, abordando complejos sistemas de interacción y regulación fisiológica de forma más detallada.

Con el paso del tiempo, se han ido perfeccionando los instrumentos y técnicas moleculares para la experimentación e investigación de los procesos biológicos de los seres vivos y gracias al avance tecnológico al que el desarrollo de esta área del conocimiento está ligado, ha permitido una mayor precisión a la hora de realizar observaciones y plantear hipótesis sobre el fenómeno estudiado. Por ello resulta pertinente, someter a la amplia variedad de investigaciones realizadas en anuros durante la segunda mitad del siglo XX, a un proceso de evaluación, donde se identifiquen nuevos hallazgos, utilizando metodologías recientes que permitan enfocar la investigación desde un punto de vista actual, con el fin de explorar las posibilidades y aplicaciones de los mecanismos adaptativos particulares de este orden.

\section{AgRadeCIMIENTOS}

A nuestros familiares y amigos por el apoyo y la motivación para emprender nuestro camino en la ciencia, a la Pontificia Universidad Javeriana por brindarnos las herramientas que hicieron posible la elaboración de este trabajo.

\section{REFERENCIAS}

Akiyoshi, H. \& Inoue, A. M. (2012). Comparative histological study of hepatic architecture in the three orders of amphibian livers. Comparative Hepatology, 11(1), 2-9. https://doi. org/10.1186/1476-5926-11-2

Amaya, E., Offield, M. F. \& Grainger, R. M. (1998). Frog genetics: Xenopus tropicalis jumps into the future. Trends in Genetics, 14(7), 253-255. https://doi.org/10.1016/S01689525(98)01506-6

Antoniazzi, C. E., López, J.A., Duré, M.\& Falico, D. A. (2013). Alimentación de dos especies de anfibios (Anura: Hylidae) en la estación de bajas temperaturas y su relación con la acumulación de energía en Santa Fe, Argentina. Revista de Biología Tropical, 61(2), 875-886.

Assis, V. R., Gardner, S. T., Smith, K. M., Gomes, F. R. \& Mendonça, M. (2020). T. Stress and immunity: Field comparisons among populations of invasive cane toads in Florida. JEZ-A Ecological and Integrative Physiology, 163, 265-271. https://doi.org/10.1002/jez.2389

Balinsky, J. (1981). Adaptation of nitrogen metabolism to hyperosmotic environment in Amphibia. J. Exp. Zool. 215(3), 335-350. https://doi.org/10.1002/jez.1402150311

Balinsky, J. B., Dicker, S. E \& Elliott, A. B. (1972). The effect of long-term adaptation to different levels of salinity on urea synthesis and tissue amino acid concentrations in $\mathrm{Ra}$ cancrivora. Comp. Biochem. Physiol, 43E, 71-82 .

Balls, M., Clothier, R. H. , Rowles J. M., Kiteley, N. A. \& Bennett, G. W. (1985). TRH distribution, levels, and significance during the development of Xenopus laevis. In Metamorphosis (eds. Balls, M. \& Bownes, M.) 260-272 (Clarendon Press, Oxford).

Baxter, C. F. \& Ortiz, C. L. (1966). Amino acids and the maintenance of osmotic equilibrium in brain tissue. Life Sci., 5, 2321-2329 https://doi.org/10.1016/00243205(66)90069-5

Bevier, C. (1997). Utilization of energy substrates during calling activity in tropical frogs. Behav. Ecol. Sociobiol., 41, 343-352. https://doi.org/10.1007/s002650050394

Bold, H. C \& Wynne, M. J. (1985). Introduction to the Algae: Structure and Reproduction. (Prentice-Hall, Michigan).

Boto, L. (2010). Horizontal gene transfer in evolution: facts and challenges. Proceedings of the Royal Society B: BiologicalSciences, 277, 819-827. https://doi.org/10.1098/ rspb.2009.1679

Brown, G. P., Shilton, C., Phillips, B. L. \& Shine, R. (2007). Invasion, stress, and spinal arthritis in cane toads. PNAS, 104, 17698-17700. https://doi.org/10.1073/pnas.0705057104

Carvalho, J., Gomes, F. \& Navas. (2008). Energy substrate utilization during nightly vocal activity in three species of Scinax (Anura/Hylidae). Journal of Comparative Physiology B, 178(4), 447-456 https://doi.org/10.1007/ s00360-007-0236-6

Cea-Calvo, L., Moreno, B., Monereo, S., Gil-Guillén, V., Lozano, J. V., Martí-Canales, J. C., Llisterri, J. L., Aznar, J., Gonzáles-Esteban, J. \& Redón, J. (2008). Prevalencia de sobrepeso y obesidad en población española de 60 años o más y factores relacionados. Estudio PREV-ICTUS. Medicina Clínica, 131(6), 205-210. https://doi.org/10.1157/13124609

Ceballos, N. R. (1999). Biosíntesis de aldosterona y su regulación en Bufo arenarum (Amphibia,Anura) (Doctoral dissertation, Universidad de Buenos Aires. Facultad de Ciencias Exactas y Naturales).

Ceballos, N. R. \& Lantos, C. P. (1992). Corticoidogenesis in Bufo arenarum $\mathrm{H}$. In vitro biosynthesis of pregnenolone and corticosterone metabolites at low temperature incubations. Gen. Comp. Endocrinol., 88, 132-139 https:// doi.org/10.1042/bj2920143

Ceballos, N. R., Cozza, E. N. \& Lantos, C. P. (1983). Corticoidogenesis in $B$. arenarum $\mathrm{H}$. I. In vitro biosynthesis of $3 \mathrm{H}$-pregnenolone and $3 \mathrm{H}$-corticosterone metabolites and of endogenous 3-oxo-4-ene intennediates at $28 \mathrm{C}$ and 37 
C. Gen. Comp. Endocrinol., 51, 138-147

Chong, R. A. \& Mueller, R. L. (2013). Low metabolic rates in salamanders are correlated with weak selective constraints on mitochondrial genes. Evolution: International Journal of Organic Evolution, 67(3), 894-899. https://doi.org/10.1111/ j.1558-5646.2012.01830.x

Citadini, J. M., Brandt, R., Williams, C. R. \& Gomes, F. R. (2018). Evolution of morphology and locomotor performance in anurans: relationships with microhabitat diversification. $J$. Evol.Biol.,31,371-381.https://doi.org/10.1111/jeb.13228

Cocroft, R. B. \& Ryan, M. J. (1995). Patterns of advertisement call evolution in toads and chorus frogs. Animal Behaviour, 49(2), 283-303 https://doi.org/10.1006/anbe.1995.0043

Colley, L., W. C. Rowe, A. K. Huggins, A. B. Elliott, \& S. E. (1972).Dicker The effect of short-term changes in the external salinity on the levels of the non-protein nitrogen- ous compounds and the ornithine urea cycle enzymes in Rana cancrivora. Comp. Biochem. Physiol., 41B, 307-322.

Coppo, J. A., Mussart, N. B., Fioranelli, S. A., Barboza, N. N. \& Koza, G. A. (2005).Variaciones fisiológicas atribuibles al crecimiento, alimentación y temperatura ambiental en sangre de Rana catesbeiana (Shaw, 1802). Revista Veterinaria, 16(2), 74-83.

Cossins, A. R. \& Prosser, C. L. (1978). Evolutionary adaptation of membranes to temperature. PNAS, 75, 2040-2043 https:// doi.org/10.1073/pnas.75.4.2040

Costa, J. (2004). Reacción en cadena de la polimerasa (PCR) a tiempo real. Enfermedades infecciosas y microbiología clínica, 22(5), 299-305.

Costanzo J. P., Wright, M. F. \& Lee, R. E. (1992). Freeze tolerance as an overwintering adaptation in Cope's grey treefrog (Hylachrysoscelis). Copeia, 1992(2), 565-569. https://doi.org/10.2307/1446222

Costanzo, J. P., Lee, R. E. \& Wright, M. F. (1991). Effect of cooling rate on the survival of frozen wood frogs, Rana sylvatica. J. Comp. Physiol. B, 161(3), 225-229. https:// doi.org/10.1007/BF00262302

Daily J. W., Spande T. F. \& Garraffo, H. M. (2005). Alkaloids from Amphibian Skin:A Tabulation of Over Eight Hundred Compounds. Journal of Natural Products, 68(10), 1556$1575 \mathrm{https}: / /$ doi.org/10.1021/np0580560

Daily J. W, Myers C. W. \& Whittaker, N. (1987). Further classification of skin alkaloids from neotropical poison frogs (Dendrobatidae), with a general survey of toxic/noxious substances in the amphibia. Toxicon, 25(10), 1023-95 https:// doi.org/10.1016/0041-0101(87)90265-0

Dapper, A. L., Baugh, A. T. \& Ryan, M. J. (2011).The sounds of silence as an alarm cue in túngara frogs, Physalaemus pustulosus. Biotropica, 43(3), 380-385 https://doi. org/10.1111/j.1744-7429.2010.00707.x

Darst, C. R., Menéndez Guerrero, P. A., Coloma, L. A. \& Cannatella, D.C.(2005). Evolution of dietary specialization and chemical defense in poison frogs (Dendrobatidae): a comparative analysis. The American Naturalist, 165(1),
56-69 https://doi.org/10.1086/426599

Denver, R. J. (2009). Structural and functional evolution of vertebrate neuroendocrine. Ann. N. Y.Acad. Sci., 1163, 1-16.

Dickhoff, W. W. (1993). Hormones, metamorphosis, and smolting In The endocrinology of growth, development, and metabolism in vertebrates (eds. Schreibman, M. P., Scanes, C. G., Pang, P. K. T.) 519-540. (Academic Press, New York).

Dinsmore, S. C. \& Swanson, D. L. (2008). Temporal patterns of tissue glycogen, glucose, and glycogen phosphorylase activity prior to hibernation in freeze-tolerant chorus frogs, Pseudacristriseriata. Can. J. Zool., 86, 1095-1100. https:// doi.org/10.1139/Z08-088

Eckert, R., Randall, D. \& Augustine, G. (1992). Fisiología Animal (Interamericana, Madrid).

Federico, L. (2011). La cloricia en anuros, análisis histórico de un carácter sistemático. Scientiae Studia, 9(4), 777-789 https://doi.org/10.1590/S1678-31662011000400003

Fernández, A. H. \& Camargo, C. D. B. (2011). El lenguaje y el genoma humano: nociones básicas para los especialistas en fonoaudiología. Revista Internacional de Investigación en Ciencias Sociales, 7(2), 161:

Fischer von Waldheim, G. F. (1813). Zoognosia tabulis synopticis illustrata: in usum praelectionum Academiae imperialis médico-chirugicae mosquensis edita. Typis Nicolai S. Vsevolozsky

Flores Soto, M. E. \& Segura Torres, J. E. (2005). Estructura y función de los receptores acetilcolina de tipo muscarínico y nicotínico. Rev. Mex. Neuroci., 6(4), 315-326.

Frost, D. R. (2021). Amphibian Species of the World: an Online Reference. Version 6.1 (accessed 6 February 2021). Electronic Database accessible at https:// amphibiansoftheworld.amnh.org/index.php. American Museum of Natural History, New York, USA. https://doi. org/10.5531/db.vz.0001

Gerhardt, H.C. (1994). The evolution of vocalization in frogs and toads. Annual Review in Ecology and Systematics, 25, 293324. https://doi.org/10.1146/annurev.es.25.110194.001453

Glazier, D. S. (2005). Más allá de la «ley de 3/4 potencias»: variación en la escala intra e interespecífica de la tasa metabólica en animales. Revisiones Biológicas, 80(4), 611-662.

Gómez Fernández, D., Castaño, S., Fierro, L., Armbrecht, I. \& Asencio-Santofimio, H. (2013). Análisis Trófico De Andinobates Minutus (Anura: Dendrobatidae) EnUnBosque Húmedo Tropical De La Isla La Palma, Colombia: Diet of Andinobates minutus (Anura: Dendrobatidae) in a tropical rainforest from La Palma island, Colombia. Caldasia, 35(2), 325-332

Gordon, M. S. (1965). Intracellular osmoregulation in skeletal muscle during salinity adaptation in two species of toads. The Biological Bulletin, 128(2), 18-229 https://doi. org/10.2307/1539551

Grafe, U. (1997). Use of metabolic substrates in the gray treefrog 
Hyla versicolor: Implications for calling behavior. Cop., 1997, 356-362.

Grundy, J. E. \& Storey, K. B. (1994). Urea and salt effects on enzymes from estivating and non-estivating amphibians. Molecular and Cellular Biochemistry, 131(1), 9-17. https:// doi.org/10.1007/BF01075719

Gutiérrez, K., Morales, R. \& Pino, J.(2018). Ranas dardo venenosas (Dendrobatidae) y su importancia en la bioprospección de moléculas bioactivas en los últimos tiempos: una revisión. Revista de Iniciación Científica, 4, 43-47 https://doi.org/10.33412/rev-ric.v4.0.1818

Handler, J., Petersen, M. \& Orloff, J. (1966). Effect of metabolic inhibitors on the response of the toad bladder to vasopressin. American Journal of Physiology-Legacy Content, 211(5), 1175-1180. https://doi.org/10.1152/ ajplegacy.1966.211.5.1175

Hanke, W. \& Neumann, U. (1972). Carbohydrate metabolism in Amphibia. General And Comparative Endocrinology, 3, 198-208. https://doi.org/10.1016/0016-6480(72)90149-9

Hart, J. S. (1957). Climatic and temperature induced changes in the energetics of homeotherms. Rev. Can. Biol., 16, 166-174.

Heyer, W. R., García-López, J. M. \& Cardoso, A. J. (1996). Advertisement call variation in the Leptodactylus mystaceus species complex (Amphibia: Leptodactylidae) with a description of a new sibling species. Amphibia-Reptilia, 17(1), 7-31

Jolivet-Jaudet, G. \& Leloup-Hatey, J. (1984). Variations in aldosterone and corticosterone plasma levels during metamorphosis in Xenopus laevis tadpoles. Gen. Comp. Endocrinol., 56, 59-65. https://doi.org/10.1016/00166480(84)90061-3

Kasai, R., Kitajima, Y., Martin, C. E., Nozawa, Y., Skn’ver, L. \& Thompson Jr., G.A. (1976). Molecular control of membrane properties dung temperature acclimation. Membrane fluidity regulation of fatty acid desaturase action. Biochemistry, 15, 5228-5233 https://doi.org/10.1021/bi00669a005

Kikuyama, S., Suzuki, M. R. \& Iwamuro, S. (1986). Elevation of plasma aldosterone levels of tadpoles at metamorphic climax. Gen. Comp. Endocrinol., 63, 186-190. https://doi. org/10.1016/0016-6480(86)90155-3

King, J. A. \& Milla, R. P. (1981). TRH, GH-RIH, and LH-RH in metamorphosing Xenopus laevis. Gen. Comp. Endocrinol., 44, 20-27.https://doi.org/10.1016/0016-6480(81)90351-8

Krane, C. M. \& Goldstein, D. L. (2007). Comparative functional analysis of aquaporins/glyceroporins in mammals and anurans. Mamm. Genome, 18(6), 452-462. DOI: 10.1007/ s00335-007-9041-5

Kupferberg, S. J. (1997). The role of larval diet in anuran metamorphosis. American Zoologist, 37(2), 146-159. https://doi.org/10.1093/icb/37.2.146

Labra, F. A., Marquet, P. A. \& Bozinovic, F. (2007). Scaling metabolic rate fluctuations. PNAS, 104(26), 10900-10903. https://doi.org/10.1073/pnas.0704108104

Layne, J. R., Jr. \& Lee, R. E., Jr. (1987). Freeze tolerance and the dynamics of ice formation in wood frogs (Rana sylvatica) from southern Ohio. Can. J. Zool., 65(8), 2062-2065. https://doi.org/10.1139/z87-315

Lighton, J. R. (2008). Measuring metabolic rates: a manual for scientists. (Oxford, Oxford University Press.

Llewellyn, D., Thompson, M. B., Brown, G. P., Phillips, B. L. \& Shine, R. (2012). Reduced investment in immune function in invasion-front populations of the cane toad (Rhinella marina) in Australia. Biological Invasions, 14, 999-1008. https://doi.org/10.1007/s10530-011-0135-3

López, J. A., Scarabotti, P. A. \& Ghirardi, R. (2011). Seasonal patterns of abundance and recruitment in an amphibian assemblage from the Paraná River floodplain. Interciencia, 36, 538-544.

Ma, T., Yang, B. \& Verkman, A. S. (1996). cDNA cloning of a functional water channel from toad urinary bladder epithelium. Am. J. Physiol., 271(5), C1699-C1704. DOI: 10.1152/ajpcell.1996.271.5.C1699

MacDonald, J. A., Degenhardt, T., Baynes, J. W. \& Storey, K. B. (2009). Glycation of wood frog (Rana sylvatica) hemoglobin and blood proteins: in vivo and in vitro studies. Cryobiology, 59(2), 223-225.

Martínez Augustin, O. \& Martínez de Victoria, E. (2006). Proteínas y péptidos en nutrición enteral. Nutrición Hospitalaria, 21, 01-14

McKee, T. \& McKee, J. R. ( 2014). Bioquímica: las bases moleculares de la vida (McGrawHill, Philadelphia).

McNabb, R. A. (1969). The effects of thyroxine on nitrogen metabolism in the leopard frog, Rana pipiens. General and Comparative Endocrinology, 13(3), 430-438 . https://doi. org/10.1016/0016-6480(69)90266-4

Milner, A. R. (1990). The radiations of temnospondyl amphibians. In Major Evolutionary Radiations (eds. Taylor, P. D. \& Larwood, G. P.) 321-349. (Systematics Association, Oxford, 1990).

Nespolo, R. F., Figueroa, J. \& Solano-Iguaran, J. J. (2017). Studying the evolutionary significance of thermal adaptation in ectotherms: The diversification of amphibians' energetics. Journal of Thermal Biology, 68, Part A, 5-13. https://doi. org/10.1016/j.jtherbio.2016.11.014

Piezzi, R. S. \& Burgos, M. H. (1968). The toad adrenal gland. 1. Cortical cells dun'ng summer and winter. Gen. Comp. Endocrinol., 10, 344-354 https://doi.org/10.1016/00166480(68)90044-0

Pough, F.H. (1980). The advantages of ectothermy for tetrapods. The American Naturalist,, 115(1), 92-112. https://doi. org/10.1086/283547

Prado, C. \& Haddad, C. F. (2005). Size-fecundity relationships and reproductive investment in female frogs in the Pantanal, South-Western Brazil. The Herpetological Journal, 15(3), 181-189.

Proaño, C. Y. \& Rivera, M. (2005). La piel de las ranas: Un verdadero arsenal químico, Nuestra Ciencia, 8, 35-38

Rincón Franco, F. \& Castro-H. F. (1998). Aspectos ecológicos 
de una comunidad de Eleutherodactylus (Anura: Leptodactylidae) en un bosque de niebla del Occidente de Colombia. Caldasia, 193-202.

Rodríguez, C., Bustos, D. \& Sanabria, E. (2019). Adaptation of the Andean Toad Rhinella spinulosa (Anura: Bufonidae) at Low Temperatures: The Role of Glucose as Cryoprotectant. Physiological And Biochemical Zoology, 92(5), 473-480. https://doi.org/10.1086/705122

Rollins-Smith, L. A. (2017). Amphibian immunity-stress, disease, and climate change. $D C I, \mathbf{6 6}, 111-119$. https://doi. org/10.1016/j.dci.2016.07.002

Ruibal, R. (1962).The Adaptive Value of Bladder Water in the Toad, Bufo cognatus. Physiol. Biochem. Zool., 35(3), 218-223. DOI:10.1086/physzool.35.3.30152806

Sawant, V. A. \& Varute, A. T. (1973). Lipid changes in the tadpoles of Rana tigrina during growth and metamorphosis. Comp. Biochem. Physiol., 44B, 729-750 https://doi. org/10.1016/0305-0491(73)90223-X

Sheridan, M. A. (1994). Regulation of lipid metabolism in poikilothermic vertebrates. Comp. Biochem. Physiol. $B$, 107(4), 495-508. https://doi.org/10.1016/03050491(94)90176-7

Sheridan, M. A. \& Kao, Y. H. (1998). Regulation of metamorphosis-associated changes in the lipid metabolism of selected vertebrates. American Zoologist, 38(2), 350$368 \mathrm{https}: / /$ doi.org/10.1093/icb/38.2.350

Sinensky, M. (1974) Homeoviscous adaptation - a homeostatic process that regulates the viscosity of membrane lipids in Escherichia coli. PNAS, 71, 522-525 . https://doi. org/10.1073/pnas.71.2.522

Steiner, A. A., Petenusci, S. O., Brentegani, L. G. \& Branco, L. G. S. (2000). The importance of glucose for the freezing tolerance/intolerance of the anuran amphibians Rana catesbeiana and Bufo paracnemis. Revista Brasileira de Biologia, 60(2), 321-328. https://doi.org/10.1590/S003471082000000200017.

Suzuki, M., Hasegawa, T., Ogushi, Y. \& Tanaka, S. (2007). Amphibian aquaporins and adaptation to terrestrial environments: a review. $C B P A, \mathbf{1 4 8 ( 1 )}, 72-81$. DOI: 10.1016/j.cbpa.2006.12.021

Tata, J. R. (1999). Amphibian metamorphosis as a model for studying the developmental actions of thyroid hormone. Biochimie, 81(4),359-366https://doi.org/10.1038/cr.1998.26
Timpone, L., Gavira, R. \& Andrade, D. (2019). Effects of temperature and meal size on the postprandial metabolic response of Leptodactylus latrans (Anura, Leptodactylidae). Journal of Experimental Zoology Part A: Ecological and Integrative Physiology, 333(2), 79-87. https://doi. org $/ 10.1002 /$ jez. 2326

Urbani, E. (1962). Comparative biochemical studies on amphibian and invertebrate development. In Advances in morphogenesis (eds. Ambercombie, M. \& Brachet, J.) 61-108 (Academic Press, New York).

Vargas, F. \& Castro, F. (1999). Distribución y preferencias de microhábitat en anuros (Amphibia) en bosque maduro y áreas perturbadas en Anchicayá, Pacífico colombiano. Caldasia, 21, 95-109.

Virkki, L.V., Franke, C., Somieski, P. \& Boron, W. F.(2002). Cloning and functional characterization of a novel aquaporin from Xenopus laevis oocytes. J. Biol. Chem., 277, 40610 40616. https://doi.org/10.1074/jbc.M206157200

Wake, D. B. (1991). Homoplasy: the result of natural selection, or evidence of design limitations? The American Naturalist, 138(3), 543-567. https://doi.org/10.1086/285234

Weber, G. M., Farrar, E. S., Tom, C. K. F. \& Grau, E. G. (1994). Changes in whole-body thyroxine and triiodothyronine concentrations and total content during early development and metamorphosis of the toad Bufo marinus. Gen. Comp. Endocrinol., 94, 62-71 . https://doi.org/10.1006/ gcen.1997.6922

Wells, K. D. \& Schwartz, J. J. (2007). The behavioral ecology of anuran communication. In Hearing and sound communication in amphibians (eds. Narins, P. M., Feng, A. S. \& Fay, R. R.). 44-86 (Springer, New York, NY.). https:// doi.org/10.1007/978-0-387-47796-1_3

Wilson, J. A. (1989). Fundamentos de Fisiología Animal (Limusa, México).

Zalduegui, P. C. (1976). Metabolismo de aminoácidos (Biblioteca ETSI Agrónomos, Madrid).

Zimmerman, S. L., Frisbie, J., Goldstein, D. L., West, J., Rivera, K. \& Krane, C. M. (2007). Excretion and conservation of glycerol, and expression of aquaporins and glyceroporins, during cold acclimation in Cope's gray tree frog Hyla chrysoscelis. Am. J. Physiol. Regul. Integr. Comp. Physiol,. 292(1), R544-R555. DOI: 10.1152/ajpregu.00434.2006. 